\title{
Balloon dilation for eustachian tube dysfunction: systematic review
}

\author{
S Y HWANG ${ }^{1,2}, \mathrm{~S} \mathrm{KOK}^{1}, \mathrm{~J}_{\text {WALTON }}^{1}$ \\ ${ }^{1}$ Department of Otolaryngology and Head and Neck Surgery, Sydney Children's Hospital, Randwick, New South \\ Wales, and ${ }^{2}$ Faculty of Medicine, University of New South Wales, Kensington, Australia
}

\begin{abstract}
Background: Eustachian tube dysfunction is a disorder for which there are limited medical and surgical treatments. Recently, eustachian tube balloon dilation has been proposed as a potential solution.

Method: A systematic literature review was performed. Abstracts were selected for relevance, and pooled data analysis and qualitative analysis was conducted.

Results: Nine prospective studies, describing 713 eustachian tube balloon dilations in 474 patients (aged 18-86 years), were identified. Follow-up duration ranged from 1.5 to 18 months. Ability to perform a Valsalva manoeuvre improved from 20 to 177 out of 245 ears following eustachian tube balloon dilation and, where data were reported in terms of patient numbers, from 15 to 189 out of 210 patients. Tympanograms were classified as type A in 7 out of 141 ears pre-operatively and in 86 out of 141 ears post-operatively.

Conclusion: Prospective case series can confirm the safety of eustachian tube balloon dilation. As a potential solution for chronic eustachian tube dysfunction, further investigations are warranted to establish a higher level of evidence of efficacy.
\end{abstract}

Key words: Auditory Tube; Balloon; Dilation; Eustachian Tube

\section{Introduction}

Eustachian tube dysfunction is a physiological disorder of the eustachian tube that results in inadequate middleear ventilation, causing aural fullness and tinnitus. In addition, complications such as serous otitis media, tympanic membrane retraction and cholesteatoma can occur. ${ }^{1}$ Eustachian tube dysfunction affects around 1 per cent of adults. ${ }^{2,3}$

Current treatment modalities for eustachian tube dysfunction, which include pharmacological agents, mechanical devices and nasal surgery, can be ineffective. $^{1,2}$ Treatment may entail multiple insertions of ventilation tubes in patients with chronic eustachian tube dysfunction, leading to complications such as tympanosclerosis, chronic perforation and cholesteatoma. 4

Recently, eustachian tube balloon dilation has been researched in prospective cohort studies and is currently used 'off-label' as a potential treatment for chronic eustachian tube dysfunction., ${ }^{5,6}$ It aims to improve eustachian tube compliance and middle-ear ventilation. Its proposed mechanisms include mechanical dilation of the cartilaginous eustachian tube and initiation of histopathological changes to the mucosa that can alter the inflammatory process. ${ }^{7}$
However, eustachian tube balloon dilation is a new procedure, and the operative technique needs to be verified for its efficacy and complications. This paper systematically reviews the available evidence on eustachian tube balloon dilation for treating chronic eustachian tube dysfunction.

\section{Materials and methods}

Criteria for study eligibility

Studies were eligible for inclusion in data analysis if they were prospective (cohort or randomised), aimed to assess the effectiveness of eustachian tube balloon dilation in adults, and included outcomes of ability to perform Valsalva or Toynbee's manoeuvre and/or tympanometry results. Retrospective studies, studies that did not include one of the two aforementioned outcomes, cadaveric studies and technical studies were excluded from data analysis but included in the discussion. Published conference abstracts and case reports were excluded from this review.

\section{Literature search and study selection}

Two authors (SH and JW) independently searched Medline, PubMed and Embase databases for relevant 


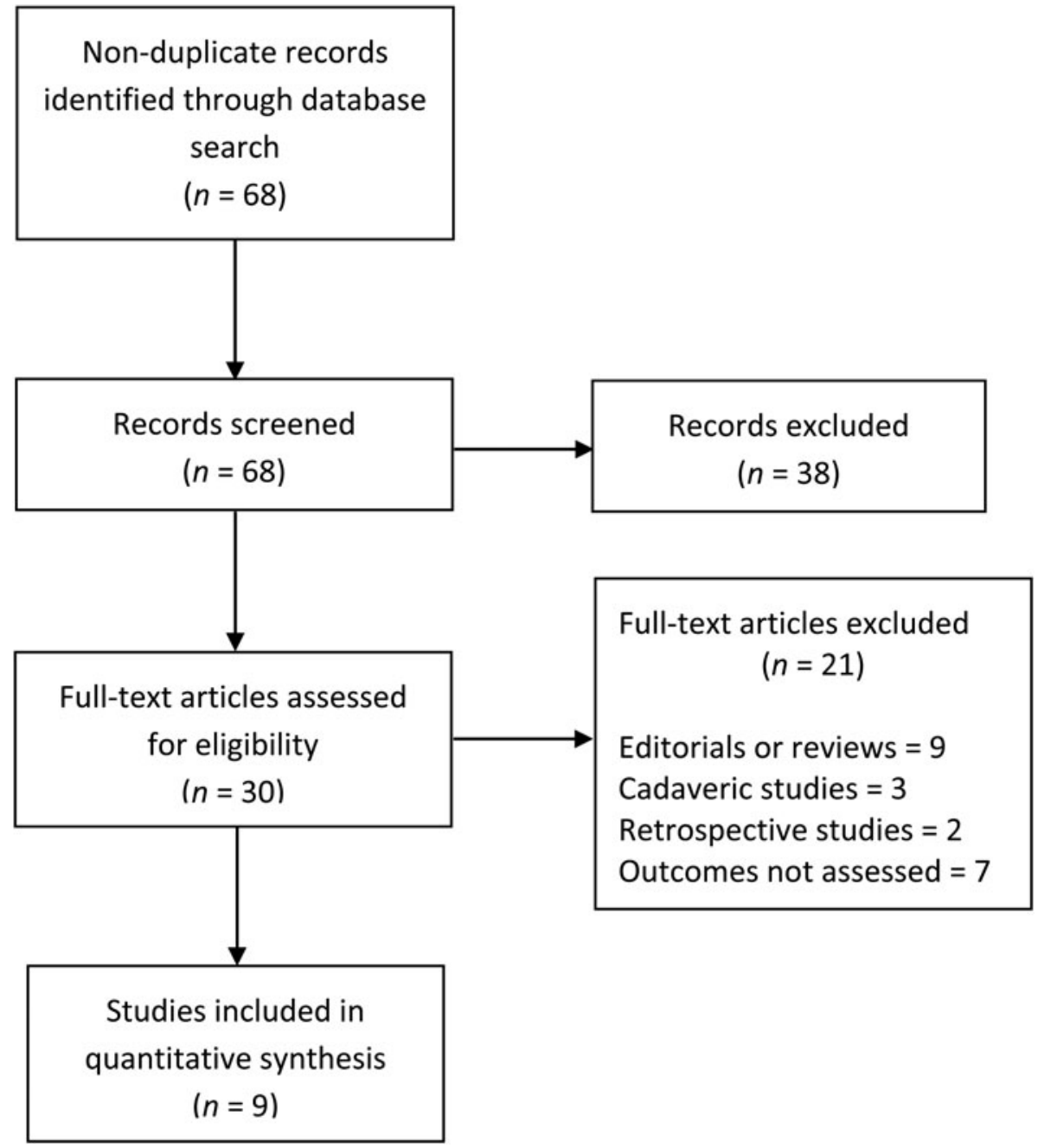

FIG. 1

Preferred Reporting Items for Systematic Reviews and Meta-Analyses ('PRISMA') flow diagram of search method.

papers published from 1950 to October 2015. No restrictions were made on language. The keywords 'eustachian tube', 'auditory tube', 'dilation', 'dilatation' and 'balloon' were used. This search was supplemented by using the 'related article' function. The search was repeated on Google Scholar to locate additional abstracts. A manual search of references of eligible manuscripts was also performed.

Studies that were eligible for inclusion in the systematic review were assessed independently and any disagreements were resolved by discussion between the two aforementioned authors. Figure 1 demonstrates the Preferred Reporting Items for Systematic Reviews and Meta-Analyses ('PRISMA') flow diagram.

\section{Data extraction and statistical analysis}

Data extraction was performed independently by two authors (SH and JW). Pooled data analysis was performed.

\section{Results}

Nine prospective studies, ${ }^{4,6,8-14}$ describing 713 eustachian tube balloon dilations in 474 patients (aged
18-86 years), were identified. Follow-up duration ranged from 1.5 to 18 months. The included studies are summarised in Tables I and II.

\section{Indications}

The indication for eustachian tube balloon dilation in all studies was chronic eustachian tube dysfunction. The method of diagnosis varied between the studies, but all included clinical history and otoscopy, with six studies using tympanometry and two studies using tubomanometry.

\section{Techniques}

Two main balloon dilation techniques were described in the literature, with five studies ${ }^{6,8,11,13,14}$ reporting the use of the Bielefeld technique (developed in Germany) and four studies ${ }^{4,9,10,12}$ using the Acclarent balloon technique (developed in North America). Both techniques were performed under general anaesthetic, with pre-operative topical decongestant applied to nasal mucosa and the eustachian tube orifice.

In the Bielefeld technique, a Storz micro-endoscope is used to visualise the insertion of a purpose-built 
TABLE I

PAPERS EVALUATING EUSTACHIAN TUBE BALLOON DILATION

\begin{tabular}{|c|c|c|c|c|c|}
\hline Authors & Year & $\begin{array}{l}\text { Pt age (mean } \\
\text { (range); years) }\end{array}$ & Number of cases & Technique & $\begin{array}{l}\text { Mean follow-up } \\
\text { time (months) }\end{array}$ \\
\hline Ockermann et al. ${ }^{6}$ & 2010 & $44.1(21-81)$ & 13 ETBDs in 8 pts & Bielefeld catheter & 2 \\
\hline Poe et al. ${ }^{10}$ & 2011 & $51.8(33-76)$ & 11 ETBDs in $11 \mathrm{pts}$ & Acclarent catheter & 7 (median) \\
\hline Catalano et al. ${ }^{4}$ & 2012 & $45(18-73)$ & 100 ETBDs in $70 \mathrm{pts}$ & Acclarent catheter & 7 \\
\hline McCoul \& Anand ${ }^{9}$ & 2012 & $55.1(\mathrm{NR})$ & 35 ETBDs in 22 pts & Acclarent catheter & 10 (median) \\
\hline Jurkiewicz et al. ${ }^{8}$ & 2013 & $45.8(23-61)$ & 7 ETBDs in 4 pts & Bielefeld catheter & 1.5 \\
\hline Schroder et al. ${ }^{11}$ & 2013 & $54(18-86)$ & 135 ETBDs in $78 \mathrm{pts}$ & Bielefeld catheter & 12 \\
\hline Tisch et al. ${ }^{13}$ & 2013 & $46(18-74)$ & 320 ETBDs in 210 pts & Bielefeld catheter & 3 \\
\hline Wanscher \& Svane-Knudsen ${ }^{14}$ & 2014 & $45(20-74)$ & 50 ETBDs in $34 \mathrm{pts}$ & Bielefeld catheter & 2 \\
\hline Silvola et al. $^{12}$ & 2014 & $48(15-38)$ & 41 ETBDs in 37 pts & Acclarent catheter & 18 \\
\hline
\end{tabular}

$\mathrm{Pt}=$ patient; $\mathrm{ETBD}=$ eustachian tube balloon dilation; $\mathrm{NR}=$ not reported

\begin{tabular}{lccccc}
\multicolumn{3}{c}{ TABLE II } \\
RESUlTS OF EUSTACHIAN TUBE BALLOON DILATION
\end{tabular}

* Results reported as numbers of patients not individual eustachian tubes. Pre-op = pre-operative; post-op = post-operative; $\mathrm{N} / \mathrm{A}=$ not applicable

Bielefeld balloon catheter into the eustachian tube. The balloon is inflated to a pressure of 10 bars for 2 minutes, with the aim of inflating the balloon to a size of $3 \times 20 \mathrm{~mm}$.

In the Acclarent balloon technique, a 30- or a 45-degree, $4 \mathrm{~mm}$ Hopkins rod is used to visualise the insertion of an angled 70-degree catheter into the eustachian tube. Balloon sizes, pressures and duration of inflation vary between the papers that use this technique. These include a $5 \times 16 \mathrm{~mm}$ balloon inflated to 8 atm for 30 seconds, ${ }^{4}$ a $5 \times 24 \mathrm{~mm}$ or $7 \times 24 \mathrm{~mm}$ balloon (adjusted for individual patients) inflated to $10 \mathrm{~atm}$ for 2 minutes, ${ }^{9}$ or a $7 \times 16 \mathrm{~mm}$ balloon inflated to $12 \mathrm{~atm}$ for 1 minute. ${ }^{10,12}$

\section{Valsalva}

The ability to perform a Valsalva or Toynbee's manoeuvre pre- and post-eustachian tube balloon dilation was reported in seven studies. This improved from 20 ( 8 per cent) to 177 ( 72 per cent) out of 245 ears following eustachian tube balloon dilation, $6,8,10-12,14$ and, where data were reported in terms of patient numbers, from 15 (7 per cent) to 189 (90 per cent) out of 210 patients. $^{13}$

\section{Tympanometry}

Tympanometry results were reported in six studies. ${ }^{8-12,14}$ Tympanograms were classified as type A in 7 out of 141 ears ( 5 per cent) pre-operatively, and this improved to 86 out of 141 ears (61 per cent) post-operatively. Twelve of these classifications (9 per cent) were because of grommets being removed and/or tympanic membrane perforations healing.

\section{Tubomanometry}

Tubomanometry was performed pre-operatively in two studies, ${ }^{6,11}$ but no post-operative data were provided. Tubomanometry was mainly used as an adjunct to aid in the diagnosis of eustachian tube dysfunction.

\section{Quality of life}

One study ${ }^{9}$ used the 7-item Eustachian Tube Dysfunction Questionnaire and the 22-item Sino-Nasal Outcome Test (SNOT-22) to evaluate improvement in symptoms and quality of life pre- and post-eustachian tube balloon dilation.

There were statistically significant improvements in both measures: the 7-item Eustachian Tube Dysfunction Questionnaire pre-operative mean score of 4.5 decreased to 2.8 at 6 months $(p<0.001)$, and the SNOT-22 pre-operative mean score decreased from 51.4 to 30 at 6 months $(p=0.001)$.

\section{Complications}

All studies documented complications. Four studies $^{6,8,9,12}$ reported no eustachian tube balloon dilation related complications and one study ${ }^{11}$ reported only minor epistaxis. More serious complications included two cases of self-resolving subcutaneous emphysema in two separate studies. ${ }^{4,13}$

Complications associated with a learning curve were described by Wanscher and Svane-Knudsen, ${ }^{14}$ who reported 3 cases of acute otitis media in the first 20 eustachian tube balloon dilations, which decreased to 1 out of 30 after 5 days of post-operative prophylactic oral antibiotics.

Poe et al. ${ }^{10}$ reported 5 cases of minor mucosal lacerations in 11 eustachian tube balloon dilations 
and 1 case of $\mathrm{C} 6-\mathrm{C} 7$ contralateral radiculopathy, the latter of which was thought to be caused by operative positioning.

There were no reported cases of carotid artery injury or patulous eustachian tube in any of the papers reviewed.

\section{Discussion}

The eustachian tube in adults is approximately $37.5 \mathrm{~mm}$ long, and consists of bony and cartilaginous portions, extending from the middle-ear cleft to the nasopharynx. ${ }^{15}$ It has several physiological functions, which include pressure equalisation, drainage of the middle ear and protection from the nasopharyngeal environment. ${ }^{15}$

Poor or inadequate eustachian tube function causes eustachian tube dysfunction, which is a physiological disorder that may be temporary and spontaneously resolving. ${ }^{1}$ Chronic eustachian tube dysfunction occurs when the dysfunction lasts for over three months; it is a poorly defined clinical entity, with variable diagnostic criteria based on clinical history, otoscopy and tympanogram results. ${ }^{2}$ It can be a difficult pathology to manage, with debilitating symptoms affecting quality of life; current conventional treatments may not be effective.

A recent health technology assessment found that there was minimal evidence of effectiveness for current medical and surgical interventions, including nasal decongestants, topical and systematic corticosteroids, antihistamines, mechanical devices, and nasal surgery. ${ }^{2}$ It identified only one study with a low risk of bias, a randomised, controlled trial, which found no improvement in eustachian tube dysfunction symptoms after six weeks of nasal steroids. ${ }^{16}$

In cases of chronic eustachian tube dysfunction refractory to conventional treatment, multiple insertions of ventilation tubes may be required. This can cause persistent perforation requiring dry ear precautions and/or myringoplasty. As a potential solution for this, eustachian tube balloon dilation (proposed as a treatment for chronic eustachian tube dysfunction) aims to ventilate and drain the middle ear by improving the physiological function of the eustachian tube. ${ }^{6,10}$

The initial papers by Ockermann et al. in $2010^{5,6}$ and Poe et al. in $2011^{10,17}$ focused on establishing the safety of eustachian tube balloon dilation by performing both cadaveric and clinical studies. The cadaveric studies revealed no evidence of fractures to the cartilaginous or bony lumen, and no damage to the internal carotid artery. Only minor mucosal lacerations at the eustachian tube orifice were noted. ${ }^{5,17}$

Since then, numerous prospective cohort studies have examined the role of eustachian tube balloon dilation. Pooled data analysis in this review revealed that the ability to perform a Valsalva improved from 8 per cent pre-eustachian tube balloon dilation to 72 per cent post-eustachian tube balloon dilation, while the rate of type A tympanograms increased from 5 to 61 per cent. Further statistical analysis is inappropriate because of the heterogeneity of the inclusion criteria, techniques and outcome measures in the papers included in this review.

The mechanisms by which eustachian tube balloon dilation improves eustachian tube function is an area of ongoing research, but appear to include both anatomical dilation of the cartilaginous eustachian tube and the initiation of histopathological changes. ${ }^{7} \mathrm{~A}$ recent study examining the histopathological changes associated with eustachian tube balloon dilation found that the balloon had a crushing effect on inflammatory cells within the eustachian tube mucosa while sparing the basal layer, rapidly replacing the inflamed mucosa with a fibrous scar. ${ }^{7}$

One major concern of eustachian tube balloon dilation is the theoretical risk of injury to a dehiscent carotid artery running adjacent to the bony eustachian tube; ${ }^{18}$ hence, computed tomography (CT) of the petrous temporal bones was routinely performed as a part of the pre-operative investigations. Abdel-Aziz et al. ${ }^{18}$ conducted a retrospective analysis of petrous temporal bone CT scans of 285 patients who underwent eustachian tube balloon dilation. The authors found that 24 eustachian tube balloon dilations were performed in 17 patients with carotid canal dehiscence, with no complications or technical difficulties. This suggests that routine petrous temporal bone CT is not indicated before eustachian tube balloon dilation.

Another area of concern for eustachian tube balloon dilation is its use in the paediatric population, where middle-ear disease is more prevalent ${ }^{19}$ because of a shorter, more horizontal eustachian tube, with a lumen that is less than half the size of an adult eustachian tube. ${ }^{15}$ Currently, there are 2 retrospective case series that describe eustachian tube balloon dilations in 33 children aged 5-14 years (mean, 9 years), ${ }^{19}$ and in 66 children aged 4-14 years (mean, 8 years) ${ }^{20}$ with no reported complications and an improvement in middle-ear symptoms.

Despite the aforementioned results of this review, Bluestone ${ }^{21}$ suggested, in 2014, that the efficacy of eustachian tube balloon dilation remains unverified. This is because the majority of studies have small numbers of patients, limited follow up, a weak definition of 'cure' and do not evaluate the direct effect of eustachian tube balloon dilation on eustachian tube function. This suggests that a more rigorous clinical trial is required.

Any future clinical trial on eustachian tube balloon dilation would require strict inclusion criteria and should use commonly accepted outcome measurements. The authors of this paper believe that the appropriate outcome measures should include: clinical measures of otoscopy and the ability to perform a Valsalva manoeuvre; objective measures of tympanometry, audiometry and/or tubomanometry; and patient-reported measures acquired using a verified questionnaire such as the seven-item Eustachian Tube Dysfunction Questionnaire. ${ }^{22}$

Our evaluation of the evidence for eustachian tube balloon dilation is limited by the quality of the papers 
included, as the highest level of evidence available is prospective case series. Three papers ${ }^{6,8,10}$ had less than 20 patients, and only 2 papers ${ }^{11,12}$ had an average follow-up period longer than 12 months. Also, there were significant variations in terms of the assessment of patients, indications for eustachian tube balloon dilation and assessment of outcomes.

The diagnosis, investigations and indications for eustachian tube balloon dilation were not standard across the papers. This standardisation is in part limited by the subjective clinical nature by which chronic eustachian tube dysfunction is diagnosed. Although all studies reviewed used clinical history, otoscopy and tympanometry to diagnose chronic eustachian tube dysfunction, the indications for eustachian tube balloon dilation differed. In some papers, eustachian tube dysfunction refractory to conventional treatment was required, while in others a diagnosis of chronic eustachian tube dysfunction was sufficient. In future evaluations of eustachian tube balloon dilation, diagnostic criteria that include objective measurements of tympanometry and the seven-item Eustachian Tube Dysfunction Questionnaire should be used.

In addition, the technique of eustachian tube balloon dilation differed across the nine papers, with the two main techniques being those described in the Results section. In both techniques, the target for balloon dilation is the $8-12 \mathrm{~mm}$ segment that acts as a valve within the cartilaginous eustachian tube, as this is where the physiological deficiency in chronic eustachian tube dysfunction is thought to originate. ${ }^{17}$ Hence, care is taken to not push the balloon catheter past the cartilaginous and bony isthmus, or to use a balloon size that is too large for the patient.

However, this has meant that a variety of balloon sizes and pressures have been employed, especially among those who use the Acclarent balloon catheters. No 'best' way to perform eustachian tube balloon dilation has yet been established; at the current early stages of evaluating its efficacy, the heterogeneity of techniques confounds the ability to draw conclusions.

Overall, this review found that eustachian tube balloon dilation is a procedure with a low rate of complications and may be considered for refractory chronic eustachian tube dysfunction in adults. More rigorous studies with standardised indications, techniques and outcomes are required to provide a higher level of evidence before its mainstream use. Nevertheless, the current data suggest a potential benefit of this procedure for a condition that can be difficult to manage.

\section{References}

1 Adil E, Poe D. What is the full range of medical and surgical treatments available for patients with eustachian tube dysfunction? Curr Opin Otolaryngol Head Neck Surg 2014;22:8-15

2 Norman G, Llewellyn A, Harden M, Coatesworth A, Kimberling D, Schilder A et al. Systematic review of the limited evidence base for treatments of eustachian tube dysfunction: a health technology assessment. Clin Otolaryngol 2014;39: $6-21$
3 Martino E, Di Thaden R, Krombach GA, Westhofen M. Function tests for the eustachian tube. Current knowledge [in German]. HNO 2004;52:1029-39; quiz 1040

4 Catalano PJ, Jonnalagadda S, Yu VM. Balloon catheter dilatation of eustachian tube: a preliminary study. Otol Neurotol 2012;33:1549-52

5 Ockermann T, Reineke U, Upile T, Ebmeyer J, Sudhoff HH. Balloon dilation eustachian tuboplasty: a feasibility study. Otol Neurotol 2010;31:1100-3

6 Ockermann T, Reineke U, Upile T, Ebmeyer J, Sudhoff HH. Balloon dilatation eustachian tuboplasty: a clinical study. Laryngoscope 2010;120:1411-16

7 Kivekas I, Chao WC, Faquin W, Hollowell M, Silvola J, Rasooly $\mathrm{T}$ et al. Histopathology of balloon-dilation eustachian tuboplasty. Laryngoscope 2015;125:436-41

8 Jurkiewicz D, Bien D, Szczygielski K, Kantor I. Clinical evaluation of balloon dilation eustachian tuboplasty in the eustachian tube dysfunction. Eur Arch Otorhinolaryngol 2013;270:1157-60

9 McCoul ED, Anand VK. Eustachian tube balloon dilation surgery. Int Forum Allergy Rhinol 2012;2:191-8

10 Poe DS, Silvola J, Pyykko I. Balloon dilation of the cartilaginous eustachian tube. Otolaryngol Head Neck Surg 2011;144:563-9

11 Schroder S, Reineke U, Lehmann M, Ebmeyer J, Sudhoff H. Chronic obstructive eustachian tube dysfunction in adults: long-term results of balloon eustachian tuboplasty [in German]. HNO 2013;61:142-51

12 Silvola J, Kivekas I, Poe DS. Balloon dilation of the cartilaginous portion of the eustachian tube. Otolaryngol Head Neck Surg 2014;151:125-30

13 Tisch M, Maier S, Maier H. Eustachian tube dilation using the Bielefeld balloon catheter: clinical experience with 320 interventions [in German]. HNO 2013;61:483-7

14 Wanscher JH, Svane-Knudsen V. Promising results after balloon dilatation of the eustachian tube for obstructive dysfunction. Dan Med J 2014;61:A4818

15 Cummings CW. Cummings Otolaryngology - Head and Neck Surgery, 4th edn. Philadelphia: Elsevier Mosby, 2005

16 Gluth MB, McDonald DR, Weaver AL, Bauch CD, Beatty CW, Orvidas LJ. Management of eustachian tube dysfunction with nasal steroid spray: a prospective, randomized, placebo-controlled trial. Arch Otolaryngol Head Neck Surg 2011;137:449-55

17 Poe DS, Hanna BM. Balloon dilation of the cartilaginous portion of the eustachian tube: initial safety and feasibility analysis in a cadaver model. Am J Otolaryngol 2011;32:115-23

18 Abdel-Aziz T, Schroder S, Lehmann M, Gehl HB, Ebmeyer J, Sudhoff H. Computed tomography before balloon eustachian tuboplasty-a true necessity? Otol Neurotol 2014;35:635-8

19 Jenckel F, Kappo N, Gliese A, Loewenthal M, Lorincz BB, Knecht $\mathrm{R}$ et al. Endonasal dilatation of the eustachian tube (EET) in children: feasibility and the role of tubomanometry (Esteve) in outcomes measurement. Eur Arch Otorhinolaryngol 2015;272:3677-83

20 Maier S, Tisch M, Maier H. Balloon dilation of the eustachian tube in pediatric chronic obstructive eustachian tube dysfunction patients [in German]. HNO 2015;63:686-8, 690-4, 696-7

21 Bluestone CD. Balloon dilation of the eustachian tube is indeed a "gizmo" until future research proves safety and efficacy. Otolaryngol Head Neck Surg 2014;151:374

22 McCoul ED, Anand VK, Christos PJ. Validating the clinical assessment of eustachian tube dysfunction: The Eustachian Tube Dysfunction Questionnaire (ETDQ-7). Laryngoscope 2012; 122: $1137-41$

Address for correspondence:

Dr Sang Y Hwang,

Department of Otolaryngology and Head and Neck Surgery,

Sydney Children's Hospital,

High Street,

Randwick,

NSW 2031, Australia

E-mail: syhwang15@gmail.com

Dr S Y Hwang takes responsibility for the integrity of the content of the paper

Competing interests: None declared 